VALARINI PJ; FRIGHETTO RTS; SCHIAVINATO RJ; CAMPANHOLA C; SENA MM; BALBINOT L; POPPI RJ. 2007. Análise integrada de sistemas de produção de tomateiro com base em indicadores edafobiológicos. Horticultura Brasileira 25: 060-067.

\title{
Análise integrada de sistemas de produção de tomateiro com base em indicadores edafobiológicos
}

\author{
Pedro José Valarini'; Rosa Toyoko Shiraishi Frighetto'; Ricardo José Schiavinato²; Clayton \\ Campanhola'; Marcelo Martins de Sena ${ }^{3}$; Leoberto Balbinot ${ }^{4}$; Ronei Jesus Poppi ${ }^{4}$ \\ ${ }^{1}$ Embrapa Meio Ambiente, C. Postal 69, 13820-000 Jaguariúna-SP; ${ }^{2}$ Fazenda Sula, 13.930-000 Serra Negra-SP; ${ }^{3}$ Unidade Universitária \\ de Ciências Exatas e Tecnológicas da Universidade Estadual de Goiás, BR 153 km 98, 7500-000 Anápolis-GO; ${ }^{4}$ Instituto de Química da \\ UNICAMP, C. Postal 6154, 13084-971 Campinas-SP; E-mail: valarini@ cnpma.embrapa.br
}

\section{RESUMO}

A análise integrada de indicadores edafobiológicos ligados ao manejo do solo constitui uma ferramenta importante para estimar níveis de sustentabilidade do agroecossistema, detectando-se pontos críticos para a devida correção de manejo. Essa ferramenta foi empregada na avaliação de sistemas de produção orgânica e convencional de tomate, em cultivo protegido e a campo aberto, no estado de São Paulo. Tomaram-se como referência solos de mata nativa e/ou pastagem natural, dependendo do local de estudo. Em Serra Negra, o solo sob sistema orgânico apresentou maior capacidade de campo e teor de argila dispersa mais baixo, indicativos da estabilidade dos agregados. No sistema convencional observou-se uma elevada condutividade elétrica, evidenciando a alta disponibilidade de sais solúveis. A análise de componentes principais (ACP) permitiu concluir que há maior grau de similaridade entre o solo sob sistema orgânico e aqueles das bases referenciais, com respeito aos indicadores químicos e biológicos. Constatou-se que $\mathrm{C}$ org, $\mathrm{N}$ total, polissacarídeos, FDA (hidrólise de diacetato de fluoresceína) e atividade enzimática de desidrogenase estão positivamente relacionados com o sistema orgânico, a mata nativa e a pastagem. Em contrapartida, a saturação por bases (V\%), $\mathrm{pH}$, teores de $\mathrm{Mn}, \mathrm{Mg}$ e $\mathrm{Ca}$, bem como a razão de dispersão estão inversamente relacionadas ao manejo orgânico. Já em Araraquara, os resultados da ACP distinguiram as áreas organicamente cultivadas das matas nativas, principalmente, com base nos indicadores biológicos.

Palavras-chave: qualidade do solo, análise de componentes principais, sustentabilidade, agroecossistema.

\section{ABSTRACT}

Integrated analysis of production systems of tomato with edaphobiological indicators

The integrated analysis for physical, chemical and biological parameters in soil management systems is an important tool to check the sustainability of agroecosystems, allowing to identify the main critical constraints that impair the best management conditions. This approach has been used to evaluate production of tomato in organic and conventional systems in the state of São Paulo, Brazil, comparing greenhouse and field cultivation. In both cases, native forest or permanent pasture was taken as basal reference in each of the evaluated rural establishments. The results from organic systems in Serra Negra showed higher water holding capacity (CC) and lower clay dispersion (microaggregation increase) than the conventional system, indicating more stabilized soil aggregates. In conventional systems higher electrical conductivity was observed, which can be indicative of high soluble salt availability for the plants. The principal component analysis (PCA) for the data collected in Serra Negra allowed to conclude that there is greater similarity between organic systems and basal references, considering most of the biological and chemical indicators. It was also observed that organic C, total N, polysaccharides, fluorescein diacetate hydrolysis (FDA) and dehydrogenase activity were positively related to organic systems, natural forest (M1) and pasture (P1), whereas V\%, pH, Mn, Mg, Ca and soil particle dispersion ratio were inversely related to those systems. The model of PCA for the data collected in Araraquara efficiently separated the cultivated areas from their respective native forests, based on biological indicators.

Keywords: soil quality, principal component analysis, agroecosystem, sustainability.

\section{(Recebido para publicação em 07 de dezembro; aceito em 10 de abril de 2007)}

$\mathrm{O}$ mercado mundial de produtos orgânicos cresce a uma taxa média de $10 \%$ ao ano, passando de U\$25 bilhões em 2003 para U\$31 bilhões em 2005 (Raynolds, 2004; IFOAM, 2005). Acompanhando esse movimento, a produção orgânica brasileira representada por 20 mil produtores basicamente familiares, cresce a uma taxa de $30 \%$ ao ano, ocupa uma área de $287 \mathrm{mil} \mathrm{ha,} \mathrm{re-}$ presenta quase $1,0 \%$ da área cultivada total e movimenta U\$ 190 milhões, dos quais, U\$ 160 milhões foram pela ex- portação em 2004. Mundialmente, o país figura como quarto em área cultivada e o sétimo colocado no fornecimento de produtos orgânicos para a União Européia (Planeta orgânico, 2005).

A olericultura orgânica é uma atividade produtiva que tem experimentado grande expansão no Brasil e no mundo, em decorrência do aumento significativo da demanda do mercado consumidor por alimentos mais saudáveis (Costa \& Campanhola, 1997; Campanhola \& Valarini, 2001; Diniz et al., 2006), despontando como uma excelente oportunidade para a agricultura familiar. $\mathrm{O}$ tomate (Lycopersicon esculentum Mill.) é uma das principais hortaliças cultivadas no Brasil. Segundo o IBGE (2007), estima-se que a produção brasileira de tomate (2006/2007) esteja em 3,3 milhões de t ocupando uma área de $58 \mathrm{mil}$ ha, sendo $27 \%$ da produção destinada à indústria e $73 \%$ ao consumo in natura. Dentre os estados produtores de tomate, São Paulo destaca-se em primeiro lugar, seguido por Minas Gerais, Goiás, 
Rio Grande do Sul e Rio de Janeiro (AGRIANUAL, 2006), tendo o mesmo, uma produção anual estimada de tomate “in natura" de 76 mil t (Produtos..., 2005).

A demanda crescente por alimentos conduz o foco, inevitavelmente, também, para a questão de manejo e a degradação do solo cultivado em função dos diferentes sistemas de produção agropecuários. Os monocultivos, a queima sistemática de restos culturais, o uso indiscriminado de fertilizantes minerais e de agrotóxicos, a excessiva utilização de máquinas agrícolas pesadas, práticas inadequadas de manejo do solo e da água, dentre outros aspectos da agricultura convencional, afetam significativamente a qualidade do solo, acarretando mudanças em suas propriedades físicas, químicas e biológicas (Fauci \& Dick, 1994; Valarini et al., 1999).

Base de sustentação da atividade agrícola, a qualidade do solo é definida por Doran \& Parkin (1994) como sendo "a capacidade de funcionar no ecossistema para sustentar a produtividade biológica, manter a qualidade ambiental e promover a saúde do animal e da planta". Tokeshi (1991) considerou a diversidade biológica presente no solo como um dos componentes mais importantes, em termos de formação e manutenção da estrutura física e da fertilidade, visto que dela dependem as populações de plantas superiores cultivadas e espontâneas, seus polinizadores e agentes de biocontrole de doenças e pragas.

Historicamente, apenas as propriedades físicas e químicas têm sido utilizadas como medidas da produtividade do solo em seu estado natural, sendo relacionadas ao teor de matéria orgânica, para o caso das áreas cultivadas. Entretanto, como as mudanças no teor de matéria orgânica são, em geral, lentas, a partir dos anos 90 passou-se a dar maior importância aos parâmetros edafobiológicos como indicadores mais sensíveis de alterações relacionadas ao manejo do agroecossistema (Fauci \& Dick, 1994; Dilly \& Blume, 1998). Esses últimos autores e também Frighetto \& Valarini (2000) recomendaram, além dos indicadores físicos e químicos, alguns indicadores de natureza biológica para aferir a qualidade do solo, como atividade enzimática (em desidrogenase e esterase), biomassa microbiana e polissacarídeos, ambos, em carbono.

A análise integrada desses parâmetros edafobiológicos deverá constituir-se em ferramenta importante para avaliar a sustentabilidade do agroecossistema, permitindo indicar pontos críticos associados ao manejo adotado.

Nas últimas duas décadas, a análise multivariada foi introduzida no tratamento de dados químicos, ganhando interesse e dando origem a uma nova disciplina, a quimiometria. O modelo estatístico de análise multivariada considera a correlação entre diversos parâmetros de análise, assim potencializando uma quantidade maior de informação. Sena et al. (2000, 2002) aplicaram a análise de componentes principais (ACP) em duas regiões do estado de São Paulo, em solos submetidos a manejo agrícola diferenciado. Os resultados da análise integrada (ACP) permitiram identificar os fatores mais relevantes para separação entre os diferentes tipos de manejo do solo. Também, Balbinot et al. (2003), comparando dois métodos quimiométricos: um linear ACP e outro não linear - MAO ("Mapa Auto-Organizável”), na discriminação de práticas de manejo, constataram, em ambos os casos, semelhança entre as características de solos sob sistema orgânico e aqueles de áreas não submetidas a interferências (mata nativa).

O presente trabalho teve como objetivo avaliar a qualidade do solo, em função de práticas culturais adotadas em tomate e olericultura orgânica em São Paulo, mediante a avaliação de seus atributos, incluindo a análise de componentes principais (ACP).

\section{MATERIAL E MÉTODOS}

O estudo foi conduzido, entre 2001 e 2003, em unidades de produção dedicadas ao cultivo do tomateiro sob sistemas orgânico (Org) e convencional (Conv), situadas nos municípios de Serra Negra (quatro) e Araraquara (duas), estado de São Paulo. Os sistemas foram codificados como seguem: Org1-EC (Serra Negra); Org2-EC, Org3-E1, Org3-E2 e Org3-E3 (Araraquara); Conv1-E, Conv2-EC e Conv3-C (Serra
Negra). As letras E e C significam cultivo em estufa e cultivo em campo, respectivamente.

Dentro das unidades de produção, tanto em cultivo protegido como em campo, o solo foi classificado como Argissolo Vermelho-Amarelo (AVA) textura franco-argilosa (Serra Negra) ou Latossolo Vermelho-Amarelo (LVA), textura média (Araraquara), segundo o Sistema Brasileiro de Classificação de Solos (Embrapa, 1999).

Diversas cultivares de tomateiro foram utilizadas, a saber: Débora, Carmen, Delta, Saladete, Jane, Letícia, SL2844, Andréa, Raísa, Colosso, Olímpia, Santa Clara e Bonus. A cultivar Santa Cruz foi encontrada apenas em unidades de produção orgânica.

As unidades de produção orgânica foram a Fazenda Sula (Org1-EC) em Serra Negra certificada pela Associação de Agricultura Orgânica (AAO) e o Sítio Oyafuso (Org2-EC) e a Chácara Aruã (Org3-E) em Araraquara, ambos certificados pelo Instituto Biodinâmico de Desenvolvimento (IBD). Nessas três unidades de produção, o manejo foi semelhante e caracterizado pelo plantio, tanto em estufa quanto no campo, de um número de cultivares de tomateiro, dentre as mencionadas. Desse manejo faziam parte o cultivo mínimo, a adubação verde e/ou orgânica (esterco), a cobertura do solo com vegetação espontânea roçada, com mulching de capim picado ou com plástico. Os tratos fitossanitários restringiram-se às pulverizações com caldas (bordalesa e/ou sulfocálcica), extratos vegetais diversos (nim, alho, pimenta, primavera, etc), agentes microbianos de biocontrole (Bacillus thuringiensis, Trichoderma harzianum), parasitóides (Trichograma spp.), armadilha luminosa, fungicidas caseiros a base de leite crú e urina de vaca, e biofertilizantes líquidos à base de torta de mamona $(10 \mathrm{~kg})$, cinzas de olaria $(20 \mathrm{~kg})$, farinha de osso $(5 \mathrm{~kg})$, calcáreo de conchas (1 kg). Além do tomateiro, outras culturas estavam presentes, como pimentão, pepino, cenoura, abobrinha, morango e folhosas, dependendo da unidade de produção, mostrando a característica de policultivo da agricultura orgânica. 
Os sistemas convencionais de produção de tomateiro foram representados pelos Sítios São José (Conv1-EC), São Marcos (Conv2-EC) e Santa Luzia (Conv3-C), todos em Serra Negra. O manejo das lavouras foi equivalente, com o solo preparado por meio de aração e gradagem, mantido descoberto e adubado com formulações comerciais de NPK, acrescido, quando disponível, de adubos orgânicos à base de esterco de vaca nas covas de plantio das mudas. No cultivo Conv verificou-se um espaçamento mais adensado na estufa do que no campo. O controle das pragas foi baseado no uso de agrotóxicos, incluindo fungicidas, tais como: metalaxyl + mancozeb, PCNB, benomyl, Kumulus; antibióticos (agrimicina) e inseticidas (paration metíl, Cloronicotinilo, azoxystrobin, buprofecin).

Foram coletadas 15 amostras simples de solo para compor uma amostra composta, na camada de 0-20 cm. Para as análises químicas e biológicas, foram coletadas amostras de solo deformadas, enquanto que, para as análises físicas, as amostras de solo foram semi-deformadas e indeformadas, conforme o atributo a ser determinado. Logo após a coleta, as amostras de solo para análise biológica foram acondicionadas em caixas de isopor, mantendo-se a temperatura ao redor de $10^{\circ} \mathrm{C}$, e transportadas até o laboratório. Utilizaram-se como base referencial, solos das áreas de mata nativa (M1, M2 e M3) ou de pastagem natural (P1 e P2). As amostras foram processadas e encaminhadas aos laboratórios da Embrapa Meio Ambiente (análises físicas e biológicas) e do Instituto Agronômico de Campinas (análise química). Foram aferidos os parâmetros químicos: teor de matéria orgânica (MO), carbono orgânico (C org), nitrogênio total ( $\mathrm{N}$ total), relação $\mathrm{C} / \mathrm{N}, \mathrm{pH}\left(\mathrm{CaCl}_{2}\right), \mathrm{H}+\mathrm{Al}, \mathrm{P}, \mathrm{K}, \mathrm{Ca}, \mathrm{Mg}$, soma de bases (SB), capacidade de troca catiônica (CTC), saturação por bases $(\mathrm{V} \%), \mathrm{B}, \mathrm{Cu}, \mathrm{Fe}, \mathrm{Mn}, \mathrm{Zn}$ e condutividade elétrica (CE); físicos: textura, capacidade de campo (CC), razão de dispersão (Rdis), estabilidade de agregados (EA) e ponto de murchamento permanente (PMP). As análises químicas e físicas foram baseadas em metodologias descritas em RAIJ et al., (2001) e Embrapa (1997), respectivamente. As análises biológicas seguiram metodologias descritas em Frighetto \& Valarini (2000), aferindo os parâmetros como a biomassa microbiana em carbono (BM), determinada pelo método de fumigação-extração; as atividades enzimáticas na cadeia respiratória, pela hidrólise de diacetato de fluoresceína (FDA); a atividade de desidrogenase (Desi), representativa da atividade microbiana total, analisada após adição de cloreto de trifenil tetrazólio (TTC) por espectrofotometria; e o conteúdo de carbono em polissacarídeos (Poli) foi obtido pela digestão em dicromato de potássio, após extração baseada na diferença de solubilidade ácido-base, seguida de determinação espectrofotométrica. Para as análises dos atributos biológicos foram asseguradas quatro repetições de laboratório e a comparação das médias foi realizada pelo teste de Tukey a 5\%. Para a ACP, foi utilizado o valor médio das amostras de cada unidade de produção. Esses dados foram analisados no programa MATLAB ${ }^{\mathrm{TM}}$, versão 5.2 (The Math Works, Natick, EUA), usando o pacote "PLS Toolbox", versão 2.0 (Eigenvector Technologies, Manson, EUA). Com referência a todas as análises, os dados foram previamente autoescalados (média 0 e variância 1), a fim de que as variáveis contribuíssem igualmente no modelo adotado, independentemente da escala em que foram medidas.

\section{RESULTADOS E DISCUSSÃO}

Os resultados das análises de parâmetros químicos, físicos e biológicos encontram-se nas Tabelas 1 e 2. Nos casos da Fazenda Sula, do Sítio São Marcos e do Sítio São José, foi considerada como base referencial a mata (M1) devido à sua localização vicinal e a mesma classe de solo.

Alguns nutrientes, como Ca, $\mathrm{Ke} \mathrm{Mg}$, apresentaram teores de 98,0 a 207,0; 0,9 a 16,5 e 33,0 a 44,0 mmolc. dm $^{-3}$, respectivamente, acima da média recomendada que é de 4,0 a 7,0;1,6 a 3,0 e 5,0 a 8,0 mmolc. $\mathrm{dm}^{-3}$ para tomate em sistemas convencionais segundo Raij et al. (2001), com reflexos na SB e na alta concentração de sais, verificada pela condutividade elétrica (CE), essencialmente, nas propriedades de manejo convencional. Segundo Silva et al. (2003), o solo é considerado salino quando a $\mathrm{CE}$ é superior a $4 \mathrm{dS} \cdot \mathrm{m}^{-1}$. Entretanto, plantas de tomate apresentam sensibilidade moderada entre 0,9 a 9,0 dS.m ${ }^{-1}$ (Rhoades et al., 1992). Isso decorreu da aplicação maciça de adubos minerais solúveis e da ausência de monitoramento da irrigação, provocando salinização na camada superficial do solo. Como essas áreas têm sido repetidamente cultivadas, essa salinização poderá atingir níveis fitotóxicos que venham a afetar negativamente a produtividade e favorecer a incidência de pragas. Nos sistemas orgânicos, embora o acúmulo de sais possa ocorrer eventualmente, seus efeitos seriam minimizados com a rotação de culturas e através de adubação verde e/ou orgânica. Segundo Müller \& Vizzotto (1999), nas recomendações à dessalinização do solo por drenagem mecânica, via irrigação, inclui-se a adubação orgânica para aumentar o teor de húmus estável e a CTC.

Nos sistemas orgânicos de produção, os valores de $\mathrm{pH}(5,2 \mathrm{a} 6,5), \mathrm{V} \%$ (58,2 a $88,0)$ e CTC $(75,4$ a 109,0$)$ mostraramse inferiores àqueles referenciados por Raij et al. (2001) para a maior disponibilidade e absorção pelo tomateiro em sistema convencional. De acordo com Müller \& Vizzotto (1999), de modo geral, as hortaliças produzem satisfatoriamente numa faixa de $\mathrm{pH}$ entre 5,5 e 6,5 e na ausência de alumínio trocável. Quando o $\mathrm{pH}$ encontra-se fora dessa faixa, podem ocorrer sintomas de deficiência nutricional, além de fitotoxicidade por alumínio e manganês. Entretanto, no presente trabalho (Tabela 1) para os valores determinados de $\mathrm{pH}$ entre 4,2 e 5,2 para P1 e Org1-EC, respectivamente, não se observaram sintomas de fitotoxicidade.

Em sistemas convencionais, devido ao manejo simplificado (baixa diversidade genética, ausência de rotação de culturas, excesso de revolvimento do solo e adubação química repetida), observa-se um desbalanço na relação de nutrientes no solo, capaz de induzir maior suscetibilidade do tomateiro a 
Tabela 1. Resultados das análises química, física, biológica de amostras de solo de quatro unidades de produção, com sistema orgânico ou convencional de cultivo de tomateiro (Results of the chemical, physical and biological analysis from soil samples of four production units, in the organic or conventional cultivation system of tomato). Serra Negra, Embrapa Meio Ambiente, 2001-2003.

\begin{tabular}{|c|c|c|c|c|c|c|c|c|c|}
\hline \multicolumn{3}{|c|}{ Parâmetro } & \multicolumn{7}{|c|}{ Amostra de solo } \\
\hline Sigla & Descrição & Unidade & Conv3-C & P2 & M1 & P1 & Org1-EC & Conv1-E & Conv2-EC \\
\hline M.O. & Matéria orgânica & $\mathrm{g} \cdot \mathrm{dm}^{-3}$ & 24,0 & 18,0 & 38,0 & 41,0 & 43,0 & 48,0 & 52,0 \\
\hline C org & Carbono orgânico & $\mathrm{g} \cdot \mathrm{dm}^{-3}$ & 13,3 & 10,7 & 29,1 & 30,1 & 29,4 & 15,3 & 18,3 \\
\hline N-total & Nitrogênio total & $\mathrm{mg} \cdot \mathrm{Kg}^{-1}$ & 1443,0 & 1211,0 & 3025,0 & 2472,0 & 2769,0 & 1643,0 & 1811,0 \\
\hline $\mathrm{C} / \mathrm{N}$ & Carbono/Nitrogênio & & 9,2 & 8,8 & 9,6 & 12,2 & 10,6 & 9,3 & 10,1 \\
\hline $\mathrm{pH}$ & Solução em CaCl2 & & 6,4 & 6,70 & 4,60 & 4,20 & 5,20 & 5,6 & 6,6 \\
\hline$P$ & Fósforo resina & $\mathrm{mg} \cdot \mathrm{dm}^{-3}$ & 85,0 & 110,3 & 9,0 & 12,0 & 77,0 & 668,0 & 362,0 \\
\hline K & Potássio & mmolc. $\mathrm{dm}^{-3}$ & 0,9 & 1,6 & 1,2 & 1,6 & 1,5 & 7,5 & 16,5 \\
\hline $\mathrm{Ca}$ & Cálcio & mmolc. $\mathrm{dm}^{-3}$ & 98,0 & 79,0 & 27,0 & 10,0 & 42,0 & 118,0 & 207,0 \\
\hline $\mathrm{Mg}$ & Magnésio & mmolc. $\mathrm{dm}^{-3}$ & 35,0 & 34,1 & 8,0 & 4,0 & 15,0 & 33,0 & 44,0 \\
\hline $\mathrm{H}+\mathrm{Al}$ & Ac. potencial & mmolc. $\mathrm{dm}^{-3}$ & 15,0 & 13,0 & 58,0 & 88,0 & 42,0 & 28,0 & 13,0 \\
\hline S.B. & Soma de bases & mmolc. $\mathrm{dm}^{-3}$ & 133,9 & 114,7 & 36,2 & 15,6 & 58,5 & 158,5 & 267,5 \\
\hline CTC & Cap.troca catiônica & mmolc. $\mathrm{dm}^{-3}$ & 148,9 & 127,7 & 94,2 & 103,6 & 100,5 & 186,5 & 280,8 \\
\hline V & Saturação em bases & $\%$ & 89,9 & 89,8 & 38,4 & 15,1 & 58,2 & 85,0 & 95,2 \\
\hline B & Boro & $\mathrm{mg} \cdot \mathrm{dm}^{-3}$ & 0,16 & 0,37 & 0,23 & 0,31 & 0,95 & 1,51 & 0,90 \\
\hline $\mathrm{Cu}$ & Cobre & $\mathrm{mg} \cdot \mathrm{dm}^{-3}$ & 4,9 & 4,6 & 2,2 & 1,7 & 3,1 & 21,2 & 10,2 \\
\hline $\mathrm{Fe}$ & Ferro & $\mathrm{mg} \cdot \mathrm{dm}^{-3}$ & 38,0 & 27,0 & 44,0 & 122,0 & 118,0 & 129,0 & 71,0 \\
\hline Mn & Manganês & $\mathrm{mg} \cdot \mathrm{dm}^{-3}$ & 35,1 & 24,9 & 17,9 & 3,2 & 4,8 & 49,7 & 29,6 \\
\hline $\mathrm{Zn}$ & Zinco & $\mathrm{mg} \cdot \mathrm{dm}^{-3}$ & 4,9 & 5,1 & 3,0 & 3,6 & 8,1 & 21,4 & 14,7 \\
\hline CE & Condutivid. elétrica & $\mathrm{dS} / \mathrm{m}$ & 45,6 & 11,7 & 4,5 & 7,2 & 11,2 & 18,7 & 22,3 \\
\hline EA & Estabilid. agregados & $\mathrm{mm}$ & 3,60 & 3,01 & 4,76 & 4,67 & 3,08 & 3,87 & 3,14 \\
\hline $\mathrm{CC}$ & C. campo $30 \mathrm{KPa}$ & $\mathrm{KPa}$ & 0,26 & 0,23 & 0,27 & 0,27 & 0,28 & - & - \\
\hline PMP & $\begin{array}{l}\mathrm{Pt}^{\circ} \mathrm{M} \text {. Perman.15000KPa } \\
\text { PPerPermanente }\end{array}$ & $\mathrm{KPa}$ & 0,13 & 0,10 & 0,23 & 0,23 & 0,23 & - & - \\
\hline CC & Capacidade campo & $\%$ & 18,58 & 12,70 & 28,01 & 21,81 & 21,46 & 20,16 & 22,05 \\
\hline RDis & Razão de dispersão & - & 0,26 & 0,30 & 0,05 & 0,05 & 0,08 & 0,15 & 0,18 \\
\hline \multicolumn{2}{|c|}{ Classificação textural } & - & $\begin{array}{l}\text { Franco- } \\
\text { argargil. }\end{array}$ & $\begin{array}{l}\text { Franco-- } \\
\text { argil. }\end{array}$ & Argiloso & Argiloso & Argiloso & Argiloso & Argiloso \\
\hline BMic & Biomassa microb. & $\mu g \mathrm{C} . \mathrm{gsolo}^{-1}$ & $39,5 d$ & $137,5 \mathrm{c}$ & $576,1 \mathrm{a}$ & $684,0 a$ & $231,8 b$ & $482,4 a$ & $473,2 a$ \\
\hline Poli & Polissacarídeos & $\mathrm{mg} \cdot \mathrm{gsolo}^{-1}$ & $1,8 b$ & $1,1 \mathrm{c}$ & $4,0 \mathrm{a}$ & $3,1 \mathrm{a}$ & $3,3 a$ & $2,2 b$ & $2,3 b$ \\
\hline FDA & $\begin{array}{l}\text { Hidrólise diacetato de } \\
\text { fluoresceína }\end{array}$ & $\begin{array}{l}\mu \mathrm{g} \mathrm{FDA} / \mathrm{g} \\
\text { solo/min }\end{array}$ & $0,8 c$ & $0,8 c$ & $4,3 a$ & $4,4 a$ & $2,8 b$ & $1,8 b$ & $1,6 b$ \\
\hline Desi & Ativid.desidrogenase & $\mu \mathrm{lH} / \mathrm{g}$ solo & $1,5 c$ & $1,79 c$ & $3,2 a$ & $3,2 a$ & $2,4 a b$ & $2,2 a b$ & $2,4 a b$ \\
\hline Ps & Patógeno de solo & $\begin{array}{l}\mathrm{N}^{\circ} \text { propag. } \\
\text { gsolo-1 }^{-1}\end{array}$ & $1,4 a$ & $0,8 b$ & 0,0 & $0,2 c$ & $0,4 c$ & $0,9 b$ & $1,3 \mathrm{ba}$ \\
\hline
\end{tabular}

Conv3-C=Tomate convencional, campo. Santa Luzia; P2=Pastagem; Org1-EC=Tomate orgânico, campo/estufa. Fazenda Sula; M1=Mata, P1=Pastagem; Conv1-E=Tomate convencional, estufa. Sítio São José; Conv2-EC=Tomate convencional, estufa/campo, Sítio São Marcos. Médias seguidas da mesma letra na horizontal não diferem significativamente por Tukey a 5\%. (Conv3-C=Conventional tomato, field. Santa Luzia; P2=Pasture; Org1-EC=Organic tomato, field/greenhouse. Fazenda Sula; M1=Mata, P1=Pasture; Conv1-E=Conventional tomato, greenhouse. Sítio São José; Conv2-EC=Conventional tomato, greenhouse/field, Sítio São Marcos. Average values followed by the same horizontal letter did not differ significantly in the Tukey tes at the level of 5\%).

pragas e doenças. Nos sistemas orgânicos, as doenças ocorreram, porém em níveis toleráveis, visto que o equilíbrio dos nutrientes disponíveis para as plantas foi favorecido pelo $\mathrm{pH}$ mais baixo. Especificamente para o caso do patógeno do solo, Sclerotinia sclerotiorum, causador do mofo branco em tomateiro, os resultados mostraram reduções significativas em termos de número de propágulos/g do solo no sistema orgânico comparado aos sistemas convencionais e às bases referenciais (Tabela 1). Segundo Cardoso (1990) e Valarini (1994), os níveis epidêmicos são estimulados por manejo inadequado do solo, onde os níveis de infestação de patógenos são tão elevados que 0,2 escleródios de $S$. sclerotiorum/g do solo são suficientes para inviabilizar a exploração econômica das culturas. No entanto, na presente pesquisa, não foram obtidos dados quantitativos sobre níveis de doenças e pragas da parte aérea de plantas em lavouras orgânicas ou convencionais.

Segundo Darlington (2005), as fontes diversificadas de MO, com baixa relação $\mathrm{C} / \mathrm{N}$, fornecem quantidades significativas de $\mathrm{N}$ durante sua decomposição. Pelos teores de $\mathrm{N}$ total e a relação $\mathrm{C} / \mathrm{N}$ aferidos no presente estudo, pode-se dizer que o mesmo processo ocorreu em Org1-EC, P1, M1 (Tabela 1) e M3 (Tabela 2). 
Tabela 2. Resultados das análises química, física e biológica das amostras de solo de duas unidades de produção, com sistema orgânico de cultivo de tomateiro (Results of the chemical, physical and biological analysis of soil samples of two production units, in the organic system of production). Araraquara, Embrapa Meio Ambiente, 2001-2003.

\begin{tabular}{|c|c|c|c|c|c|c|c|c|c|}
\hline \multicolumn{3}{|c|}{ Parâmetro } & \multicolumn{7}{|c|}{ Amostra de solo } \\
\hline Sigla & Descrição & Unidade & Org2-E & Org2-C & M2 & Org3-E1 & Org3-E2 & Org3-E3 & M3 \\
\hline M. O. & Matéria orgânica & $\mathrm{g} \cdot \mathrm{dm}^{-3}$ & 18,0 & 24,0 & 34,0 & 29,0 & 34,0 & 29,0 & 42,0 \\
\hline C org. & Carbono orgânico & g.dm ${ }^{-3}$ & 10,5 & 13,9 & 19,8 & 17,0 & 19,8 & 16,9 & 24,4 \\
\hline N Total & Nitrogênio total & $\mathrm{mg} / \mathrm{kg}$ & 1120,2 & 1192,1 & 1155,6 & 1264,9 & 1588,3 & 1699,9 & 3583,2 \\
\hline $\mathrm{C} / \mathrm{N}$ & Carbono/Nitrogênio & Razão C/N & 9,4 & 11,7 & 17,1 & 13,4 & 12,5 & 9,9 & 6,8 \\
\hline $\mathrm{pH}$ & Solução em CaCl2 & $\begin{array}{l}\text { Solução em } \\
\mathrm{CaCl}^{2}\end{array}$ & 6,40 & 6,50 & 3,80 & 6,40 & 5,60 & 6,30 & 5,60 \\
\hline$P$ & Fósforo resina & $\mathrm{mg} \cdot \mathrm{dm}^{-3}$ & 47,0 & 65,0 & 9,0 & 94,0 & 105,0 & 51,0 & 81,0 \\
\hline K & Potássio & mmolc. $\mathrm{dm}^{-3}$ & 2,0 & 6,0 & 1,1 & 8,1 & 6,4 & 7,5 & 1,0 \\
\hline $\mathrm{Ca}$ & Cálcio & mmolc. $\mathrm{dm}^{-3}$ & 69,0 & 51,0 & 9,0 & 52,0 & 33,0 & 35,0 & 64,0 \\
\hline $\mathrm{Mg}$ & Magnésio & mmolc. $\mathrm{dm}^{-3}$ & 25,0 & 30,0 & 2,0 & 24,0 & 12,0 & 13,0 & $<1$ \\
\hline $\mathrm{H}+\mathrm{Al}$ & Ac. potencial & mmolc. $\mathrm{dm}^{-3}$ & 13,0 & 13,0 & 72 & 19 & 24 & 34 & 18,0 \\
\hline SB & Soma de bases & mmolc. $\mathrm{dm}^{-3}$ & 96,0 & 87,0 & 12,1 & 84,1 & 51,4 & 55,5 & 65,0 \\
\hline С.T.C. & Cap.troca catiônica & mmolc. $\mathrm{dm}^{-3}$ & 109,0 & 100,0 & 84,1 & 103,1 & 75,4 & 89,5 & 83,0 \\
\hline V & Saturação em bases & $\%$ & 88,0 & 87,0 & 14,4 & 81,6 & 68,2 & 62,0 & 78,3 \\
\hline$B$ & Boro & $\mathrm{mg} \cdot \mathrm{dm}^{-3}$ & 1,02 & 0,76 & 0,26 & 1,64 & 1,59 & 4,88 & 0,26 \\
\hline $\mathrm{Cu}$ & Cobre & $\mathrm{mg} \cdot \mathrm{dm}^{-3}$ & 8,4 & 14,4 & 0,8 & 12,2 & 8,2 & 10,2 & 17,7 \\
\hline $\mathrm{Fe}$ & Ferro & $\mathrm{mg} \cdot \mathrm{dm}^{-3}$ & 26,0 & 29,0 & 120,0 & 27,0 & 77,0 & 18,0 & 114,0 \\
\hline $\mathrm{Mn}$ & Manganês & $\mathrm{mg} \cdot \mathrm{dm}^{-3}$ & 14 & 11,6 & 10 & 21,2 & 32,3 & 22,5 & 11,1 \\
\hline $\mathrm{Zn}$ & Zinco & $\mathrm{mg} \cdot \mathrm{dm}^{-3}$ & 10,9 & 14,1 & 5,0 & 16,5 & 4,0 & 5,1 & 8,6 \\
\hline CE & Condutividade elétrica & $\mathrm{dS} / \mathrm{m}$ & 6,8 & 9,6 & 2,3 & 7,1 & 3,7 & 6,7 & 6,4 \\
\hline EA & Estab. agregados & $\mathrm{mm}$ & 1,27 & 1,90 & 4,53 & 4,02 & 4,26 & 4,51 & 4,77 \\
\hline $\mathrm{CC}$ & Capacidade de campo & $\%$ & 14,53 & 15,36 & 20,63 & 15,85 & 19,30 & 20,54 & 27,62 \\
\hline RDis & Razão de dispersão & & 0,07 & 0,07 & 0,08 & 0,07 & 0,16 & 0,15 & 0,29 \\
\hline Classif. & extural & & $\begin{array}{l}\text { Franco argilo- } \\
\text { arenoso }\end{array}$ & $\begin{array}{l}\text { Franco } \\
\text { Argilo- } \\
\text { arenoso }\end{array}$ & $\begin{array}{c}\text { Franco } \\
\text { argilo- } \\
\text { arenoso }\end{array}$ & $\begin{array}{l}\text { Argilo- } \\
\text { arenoso }\end{array}$ & $\begin{array}{l}\text { Argilo- } \\
\text { arenoso }\end{array}$ & $\begin{array}{l}\text { Argilo- } \\
\text { arenoso }\end{array}$ & $\begin{array}{l}\text { Argilo- } \\
\text { arenoso }\end{array}$ \\
\hline BMic & Biomassa microbiana & $\mu \mathrm{gC}$.gsolo-1 & $148,1 \mathrm{c}$ & $187,7 \mathrm{bc}$ & $350,8 a$ & $247,3 b$ & $177,4 \mathrm{bc}$ & $224,2 \mathrm{bc}$ & $417,2 a$ \\
\hline Poli & Polissacarídeos & mg.gsolo-1 & $1,2 b$ & $1,1 b$ & $1,5 b$ & $1,6 b$ & $1,5 b$ & $1,4 b$ & $2,7 a$ \\
\hline Desi & Atividade desidrogenase & $\begin{array}{l}\mu \mathrm{lH} \text {.gsolo }{ }^{-1} \\
\mathrm{~N}^{0}\end{array}$ & $2,1 d$ & $4,4 \mathrm{~cd}$ & $8,9 b$ & $6,7 b c$ & $6,8 \mathrm{bc}$ & $4,3 \mathrm{~cd}$ & $12,0 \mathrm{a}$ \\
\hline Ps & Patógeno de solo & $\begin{array}{l}\text { propágulo } \\
\text { gsolo-1 }\end{array}$ & $0,5 a b$ & $0,3 b$ & $0,1 \mathrm{c}$ & 0,0 & $0,6 a$ & $0,7 a$ & $0,02 c$ \\
\hline
\end{tabular}

Org2-E=Tomate orgânico, Letícia, estufa; Org2-C=Tomate orgânico, SL2844, estufa Sítio Oyafuso; M2 = Mata; Org3-E1=Tomate orgânico, Jane, estufa; Org3-E2=Tomate orgânico, Saladete, estufa; Org3-E3 = Tomate orgânico, Letícia , estufa, Chácara Aruã, M3=Mata. Médias seguidas da mesma letra na horizontal não diferem significativamente por Tukey a 5\%. (Org2-E=Organic tomato, Letícia, greenhouse; Org2-C=Organic tomato; SL2844, greenhouse, Sítio Oyafuso; M2 = Mata; Org3-E1=Organic tomato, Jane, greenhouse; Org3-E2=Organic tomato, Saladete, greenhouse; Org3-E3=Organic tomato, Letícia, greenhouse, Chácara Aruã, M3=Mata. Average values followed by the same letter in the horizontal did not differ significantly in the Tukey test, 5\%).

O N total disponível, em teores mais altos nessas unidades de produção, poderia ser prejudicial à cultura do tomateiro, por tornar as plantas mais suscetíveis a pragas e agentes causais de doenças (Tokeshi,1991). Entretanto, parte desse $\mathrm{N}$ é utilizada pelos próprios microrganismos, como fonte de nutrientes durante a decomposição dessa matéria orgânica, assim, promovendo a imobilização da parte que representaria um excedente para a planta. Embora as relações C/N sejam estreitas (d"12), em ambos os sistemas de produção, indicando solos com $\mathrm{N}$ estabilizado (Mello et al., 1987), as fontes de matéria orgânica mais diversificadas podem refletir nos indicadores biológicos (Tabelas $1 \mathrm{e}$ 2) (Mäder et al., 1997). Além disso, pode-se observar que em ORG1-EC não houve depreciação do teor de C orgânico, comparativamente à mata nativa tomada como base referencial.

Quanto aos indicadores físicos, nas unidades produtivas de Serra Negra (Tabela 1), o solo sob manejo orgânico apresentou maior CC (\% em peso) em relação ao manejo convencional, indicando maior capacidade de retenção da água no solo. Entretanto, devido a clas- se textural mais argilosa do solo em P1 e Org1-EC, menor disponibilidade de água para as plantas foi observada através das diferenças mais estreitas $(0,04-$ $0,05)$ entre a $\mathrm{CC}(\mathrm{kPa})$ e o PMP $(\mathrm{kPa})$, em relação àquela no sistema Conv3-C $(0,13)$. Esses dados são importantes na definição da frequiência de irrigação (Lopes, 1989). É conhecido que os solos mais argilosos fornecem condições propícias a uma rápida compactação, especialmente se o manejo da irrigação e do preparo forem inadequados. Entretanto, esse fenômeno não foi detectado no sistema orgânico de Serra Negra, 

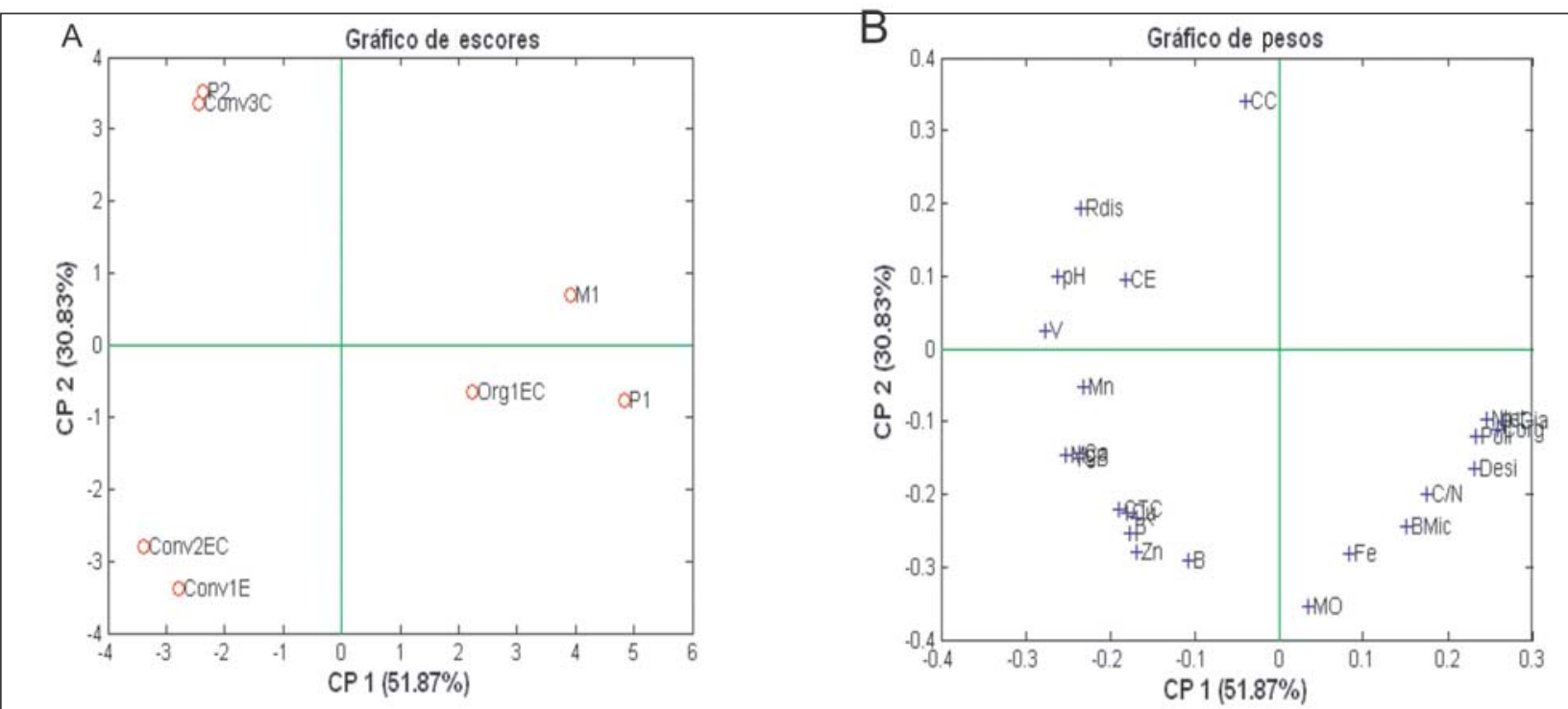

Figura 1. Resultados da ACP dos dados de Serra Negra. A) Escores de CP1 x CP2, das unidades de produção Conv1E, Conv2EC, Conv3C, Org1EC, P1, P2 e M1; B) Pesos de CP1 x CP2, dos parâmetros CC, Rdis, pH, V, CE, Mn, Mg, Ca, SB, CTC, Cu, K, P, Zn, B, MO, Fe, BMic, C/N, Desi, Poli, Ntot, Dia=FDA, Corg (Results of the ACP from the Serra Negra data. A) Escores of CP1 x CP2, from the production units Conv1E, Conv2EC, ConvC, Org1EC, P1, P2 and M1; B) Weights of CP1 x CP2, from the parameters CC, Rdis, Ph, V, CE, Mn, Mg, Ca, SB, CTC, Cu, K, P, Zn, B, Mo, Fe, BMic, C/N; Desi, Poli, Ntot, Dia+FDA) Jaguariúna, Embrapa Meio Ambiente, 2001-2003.

como os resultados mostraram através das análises de argila dispersa e dos teores de matéria orgânica. No caso dos atributos biológicos, a Tabela 1 mostra que, de um modo geral, M1 e P1 se diferenciaram dos manejos adotados nas áreas de cultivo, notadamente verificados pelos indicadores BM e FDA, seguidos de Poli e Ps, sendo que esses últimos dois diferenciaram o Org1-EC dos convencionais.

Em Araraquara (Tabela 2), os valores dos atributos: teor de $\mathrm{MO}, \mathrm{EA}, \mathrm{CC}$ e os biológicos, nas áreas M2 e M3, diferenciaram-se das áreas de cultivo (Orgs 2 e 3), mostrando que nessas últimas há necessidade de melhoria no manejo do solo em relação aos controles.

Para análise de componentes principais (ACP), englobando sistemas orgânico e convencionais, pastagem natural e mata nativa (Tabela 1), as variáveis originais são representadas por um número reduzido de combinações lineares, que retêm a maior parte da variância original (Sena et al., 2002). Os resultados da ACP confirmaram as observações anteriores sobre a similaridade entre o sistema orgânico e a mata nativa, em contraposição aos sistemas convencionais. Além disso, os gráficos de ACP (Figuras 1A e 1B) ilustram as relações entre as unidades de produção e as rela- ções entre as variáveis, permitindo uma visualização integrada dos respectivos manejos. Um modelo ACP com 2 CPs explicou $83 \%$ da variância total. A CP1 com 52\% separou o Org1, M1 e pastagem $\mathrm{P} 1$ dos demais agroecossistemas, P2 e Conv 1, 2 e 3 (Fig 1A), e a CP2 com $31 \%$, por sua vez, distinguiu o Conv3 e a P2 dos demais, Conv1 e Conv2. Demonstra-se com a Fig 1B que C orgânico, $\mathrm{N}$ total, polissacarídeos, FDA e desidrogenase sofreram influência positiva do sistema orgânico (SOrg); enquanto $\mathrm{V} \%, \mathrm{pH}, \mathrm{Mn}, \mathrm{Mg}, \mathrm{Ca}$ e razão de dispersão (Rdis) foram inversamente correlacionadas.

Os resultados da ACP de Serra Negra indicam maior preservação da qualidade do solo na área sob Org, principalmente em termos de indicadores biológicos, representando similaridade com as áreas de mata nativa (M1), as quais não sofreram ação antrópica. Além disso, detectou-se na ACP a equivalência entre pastagem (P1) e mata nativa (M1), contrastando com o sistema Conv3 que se assemelhou à pastagem vizinha (P2). Segundo Frighetto \& Valarini (2000), a área de pastagem permanente ou bem manejada com adição de resíduos no solo, resultam na melhoria da estrutura, envolvendo a formação de agregados com certo grau de estabilidade. Este fe- nômeno foi evidenciado pela presença de polissacarídeos que participam no desenvolvimento de agregados estáveis (Tabela 1).

Já a ACP de Araraquara, com duas CPs explicou $73 \%$ da variância total. A CP1 $(46,6 \%)$ separou as áreas das matas M2 e M3 das respectivas unidades produtivas, situando-se os sistemas Org2 e Org3 em posição intermediária (Fig 2A). As variáveis que contribuíram para o modelo foram: $\mathrm{N}$ total, polissacarídeos (Poli), C orgânico, teor de matéria orgânica (MO), biomassa microbiana (BMic), atividade de desidrogenase (Desi), estabilidade de agregados (EA) e capacidade de campo (CC) (Fig 2B). A CP2 com 26\%, separou os fragmentos de mata (M2 e M3) entre si, com as variáveis $\mathrm{pH}, \mathrm{SB}, \mathrm{CE}$, $\mathrm{Zn}, \mathrm{Ca}, \mathrm{Cu}, \mathrm{P}, \mathrm{e} \mathrm{V} \%$ fortemente associadas ao terceiro fragmento (M3), como indicado pelas projeções no eixo de $\mathrm{CP} 2$ (Fig 2B). Os resultados da ACP de Araraquara indicam que a qualidade do solo nas áreas de cultivo orgânico (Org2 e 3) distingue-se da mata (M3), sendo esta caracterizada pelos parâmetros: biológicos Poli, Desi e BMic; físicos CC, EA, Rdis, químicos MO e $\mathrm{N}$ total. As variáveis com altos valores em $\mathrm{CP} 1$ no gráfico de pesos estão positivamente relacionadas às matas, enquanto as va- 

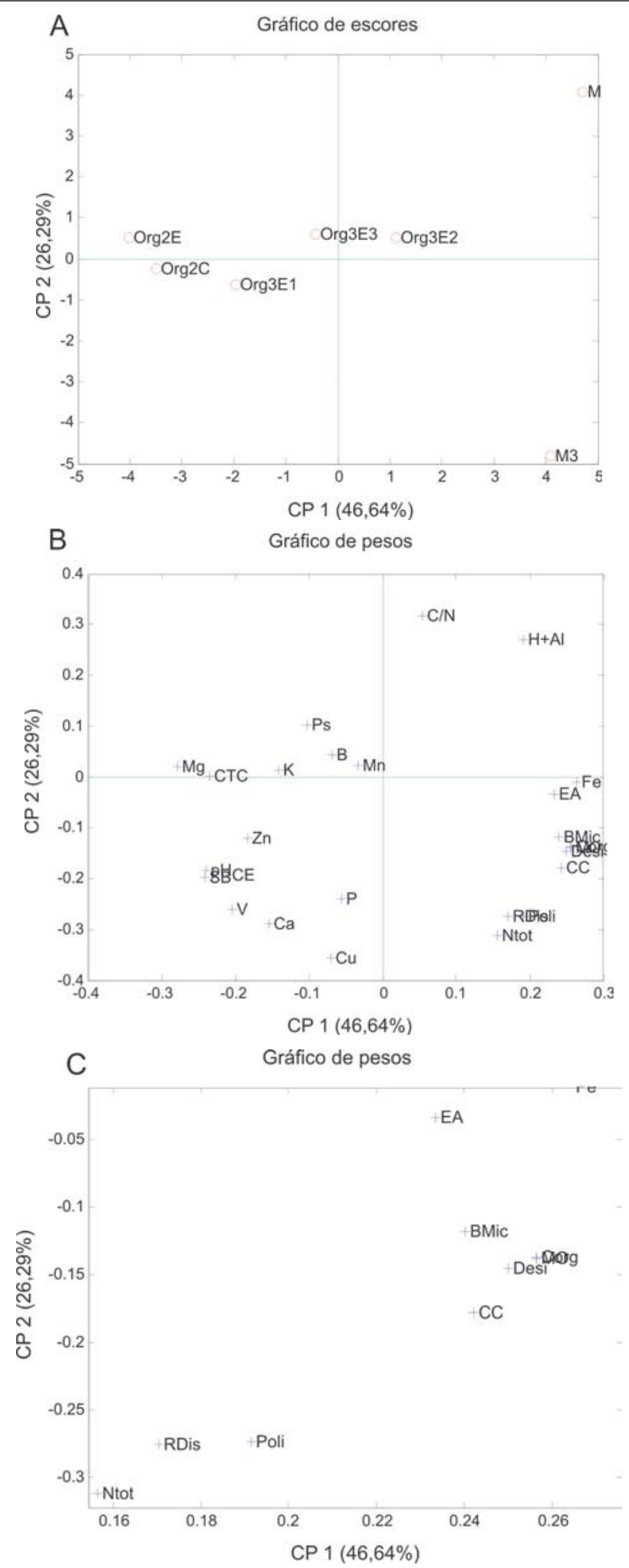

Figura 2. Resultados da ACP dos dados de Araraquara. A) Escores de CP1 x CP2, das unidades de produção Org3E1, Org3E2, Org3E3, Org2E, Org2C, M2 e M3; B) Pesos de CP1 x CP2, dos parâmetros $\mathrm{Cu}, \mathrm{Ca}$, Ntot, V, Rdis, $\mathrm{P}, \mathrm{SB}, \mathrm{CE}, \mathrm{pH}, \mathrm{CC}$, Desi., Corg, MO, Zn, BMic, EA, Fe, CTC, Mg, K, Mn, B, Poli, H+Al e C/N; C) Quadrante direito inferior do Gráfico de Pesos ampliado (Results of the ACP from the Araraquara data. A) Scors of CP1 x CP2, from the production units Org3E1, Org3E2, Org3E3, Org2E, Org2C, M2 and M3; B) Weights of CP1 x $\mathrm{CP}$ 2, from the parameters $\mathrm{Cu}, \mathrm{Ca}$, Ntot, V, Rdis, $\mathrm{P}, \mathrm{SB}, \mathrm{CE}, \mathrm{pH}, \mathrm{CC}$, Desi., Corg, MO, Zn, BMic, EA, Fe, CTC, Mg, K, Mn, B, Poli, H+Al and C/N; C) Right inferior quadrant from the graphic of amplified weights) Jaguariúna, Embrapa Meio Ambiente, 2001-2003.

riáveis com baixos valores em $\mathrm{CP} 1$ estão negativamente relacionadas às matas e positivamente às unidades de agricultura orgânica.

Finalmente, o sistema orgânico de produção de tomateiro proporciona maior diversidade microbiana no solo, disponibilidade de nutrientes, melhoria da estrutura e da fertilidade do solo e redução de inóculo de patógenos de solo em relação ao convencional, em Serra Negra. A análise integrada de atributos biológicos, químicos e físicos, utilizando a ACP, permite distinguir, com maior segurança e confiabilidade, as mudanças que ocorrem no solo em função do manejo. Os atributos edafobiológicos constituem-se em importantes indicadores da qualidade do solo.

\section{AGRADECIMENTOS}

Ao Programa de Apoio à Agricultura Familiar do CNPq pelo suporte financeiro; aos técnicos Dagmar N dos Santos Oliveira, Miriam LP Marion e Meire CS Ferrari, pelo apoio nas análises de laboratório; a Lícia Mayumi Oda, bolsista de IC do CNPq; e, em especial, aos agricultores que muito contribuíram para o sucesso da pesquisa.

\section{REFERÊNCIAS}

AGRIANUAL 2006: Anuário da agricultura brasileira. São Paulo FNP. p.473-482.

BALBINOT L; SENA MM; POPPI RJ; FRIGHETTO RTS; VALARINI PJ. 2003. Aplicação de métodos quimiométricos na discriminação de práticas de manejo de solos. In: ENCONTRO NACIONAL DE QUÍMICA ANALÍTICA, 12. São Luís. Anais... São Luís: Sociedade Brasileira de Química, QM028.

CAMPANHOLA C; VALARINI PJ. 2001. A agricultura orgânica e seu potencial para o pequeno agricultor. Cadernos de Ciência \& Tecnologia 18: 69-101.

CARDOSO JE. 1990. Fungos de solo na cultura do feijoeiro irrigado. In: FANCELLI AL. Feijão irrigado. Piracicaba: FEALQ, p.61-70.

COSTA MBB; CAMPANHOLA C. 1997. A agricultura alternativa no Estado de São Paulo. Jaguariúna: Embrapa Meio Ambiente, 63p. (Embrapa Meio Ambiente. Documentos, 7).

DARLINGTON W. Compost - A Guide for evaluating and using compost materials as soil amendments, www.soilandplantlaboratory.com, acesso em 02 maio 2005.

DILLY O; BLUME HP. 1998. Indicators to assess sustainable land use with reference to soil microbiology. Advanced GeoEcology 31: 29-36.

DINIZ LP; MAFFIA LA; DHINGRA OD; CASALI VWD; SANTOS RHS; MIZUBUTI ESG. 2006. Avaliação de produtos alternativos para controle da requeima do tomateiro. Fitopatologia Brasileira 31: 171-179. 
DORAN JW; PARKIN JB. 1994. Defining and assessing soil quality. In: DORAN JW; COLEMAN DC; BEZDICEK DF; STEWART BA. (Ed.). Defining soil quality for a sustainable environment. Madison: Soil Science Society of America, 1: 3-22. (SSSA Special Publication, 35).

EMBRAPA. 1997. Serviço Nacional de Levantamento e Conservação de Solos. Manual de métodos de análise de solo. $2^{\mathrm{a}} \mathrm{ed}$. Rio de Janeiro, (EMBRAPA-CNPS. Documentos,1). 1v. 212 p.

EMBRAPA. 1999. Sistema brasileiro de classificação de solos. Brasília: Embrapa Produção de Informação; Rio de Janeiro: Embrapa Solos, 412p.

FAUCI MF; DICK RP. 1994. Microbial biomass as an indicator of soil quality: effects of longterm management and recent soil amendments. In: DORAN JW; COLEMAN DC; BEZDICEK DF; STEWART BA. (Ed.) Defining soil quality for a sustainable environment. Madison: Soil Science Society of America. 17: 229-234. (SSSA Special Publication, 35).

FRIGHETTO RTS; VALARINI PJ. 2000. Indicadores biológicos e bioquímicos da qualidade do solo: manual técnico. Jaguariúna: Embrapa Meio Ambiente, 198p. (Embrapa Meio Ambiente. Documentos, 21).

IBGE. Sistema IBGE de recuperação automática SIDRA; Disponível em: <http//www.ibge.gov.br/ bda/prevsaf>. Acesso em 05 mar 2007.

IFOAM about IFOAM. Disponível em: <http// www.ifoam.org>. Acesso em: 14 abr. 2005.

LOPES AS. 1989. Manual de Fertilidade do Solo. São Paulo. ANDA /POTAFOS. 153p.
MÄDER P; PFIFFNER L; FLIESSBACHA; VONLÜTZOW M; MUNCH JC. 1997. Soil ecologyImpact of organic and conventional agriculture on soil biota and its significance for soil fertility. In: INTERNATIONAL CONFERENCE ON KYUSEI NATURE FARMING, 5., Bangkok, 1997. Proc... Bangkok, Asia Pacific Natural Agriculture Network. p. 24-40.

MELLO AF; BRASIL SOBRINHO MO ARZOLLA S; SILVEIRA RI; COBRA NETTO A; KIEHL J. 1987. Fertilidade do solo. 3.ed. São Paulo: Nobel. 400p.

MÜLLER JJV; VIZZOTTO VJ. 1999. Manejo do solo para produção de hortaliças em ambiente protegido. Informe Agropecuário 20: 32-35.

PLANETA ORGÂNICO. Dispon. $<$ http// www.planetaorgânico.co.br>Acesso em 15.jun. 2005.

PRODUTOS orgânicos: Brasil. Brasília, DF: Ministério da Agricultura, Pecuária e Abastecimento, 2005. 1 folder.

RAIJ B; ANDRADE JC; QUAGGIO JAA. 2001. Análise química para avaliação da fertilidade de solos tropicais. Campinas: Instituto Agronômico. 285p.

RAYNOLDS LT. 2004. The globalization of organic agro-food networks. World Development 32: 725-743.

RHOADES JD; KANDIAH A; MASHALI AM. 1992. The use of saline waters for crop production. Rome: FAO. 132p.

SENA MM; POPPI RJ; FRIGHETTO RTS; VALARINI PJ. 2000. Avaliação do uso de métodos quimiométricos em análise de solos. Química Nova 23: 547-556.
SENA MM; FRIGHETTO RTS; VALARINI PJ; TOKESHI H; POPPI RJ. 2002. Discrimination of management effects on soil parameters by using principal component analysis: a multivariate analysis case study. Soil \& Tillage Research 67: 171-181.

SILVA JBC. Cultivo de tomate para industrialização. Brasília, DF: Embrapa Hortaliças, 2003. (Embrapa Hortaliças. Sistemas de produção, 3). Disponível em:<http:// sistemasdeproducao.cnptia.embrapa.br/ FontesHTML/Tomate/TomateIndustrial/ solos.htm>. Acesso em: 19.maio.2006.

TOKESHI H. 1991. Manejo da microflora epífita no controle de doenças de plantas. In: REUNIÃO BRASILEIRA SOBRE CONTROLE BIOLÓGICO DE DOENÇAS DE PLANTAS, 4., 1991, Campinas. Anais... Jaguariúna: EMBRAPA-CNPDA. p.32-62.

VALARINI PJ. 1994. Manejo de doenças do solo em cultura de feijão sob irrigação por pivô central. In: SEMINÁRIO SOBRE PRAGAS, DOENÇAS E PLANTAS DANINHAS DO FEIJOEIRO, 5., 1994, Piracicaba. Anais. Campinas: Instituto Agronômico de Campinas. p.59-74.

VALARINI PJ; FRIGHETTO RTS; TOKESHI H; SCRAMIN S; SILVEIRAAPD; VALARINI MJ; OLIVEIRA DA. 1999. Physico-chemical and microbiological properties of soil as affected by irrigated agricultural systems. In: FOGUELMAN D; LOCKERETZ W. (Ed.) INTERNATIONAL IFOAM SCIENTIFIC CONFERENCE, 12., 1999, Mar del Plata: Proceedings... Mar Del Plata: IFOAM, p.165-171. 